\title{
Development of the Forest Engineering Experiment Teaching Website Based on ASP
}

\author{
Li Chen , Xiao Sheng-ling*, Yang Xue-chun \\ Northeast Forestry University, China \\ * Corresponding Author: shenglingxiao@yahoo.com.cn
}

\begin{abstract}
This paper built the Forest Engineering experiment teaching website based on ASP technology. It analyzed the working principle of ASP, explored the Access database connection method and the approach of SQL command operating database. There was an example of the administrator login section in order to introduce how to realize the function. Supported by the network and multimedia technologies, that developed an unlimited, independent, open space for the learner. It enhanced effectively the traditional experiment teaching methods.
\end{abstract}

Keywords: ASP, website, experiment teaching methods

\section{Working principle of ASP}

The full name of ASP is Active Server Pages, it is the development environment of the web server-side, it can create and execute dynamic, interactive, highperformance web service programs[1]. It is able to combine of HTML, scripting

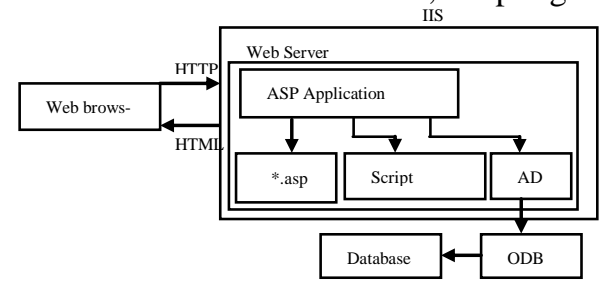

Fig. 1: Working principle of ASP language and ActiveX component to form an application program which can run on the server, and the standard HTML page is sent to the client browser. The working principle of ASP is shown in Fig.1[2].

\section{System module design}

The whole website is divided into 6 modules including homepage, laboratory situation, teaching staff, experimental teaching, scientific research, and online answering, in addition, the entire website is managed by independent background management module. Several main modules and the structure frameworks are described as follows[3-4].

\subsection{Homepage}

Homepage includes three parts they are the forest engineering experiment teaching center situation, laboratory news and notification \& announce-ment. The structure framework of laboratory news module is shown as Fig. 2[5].

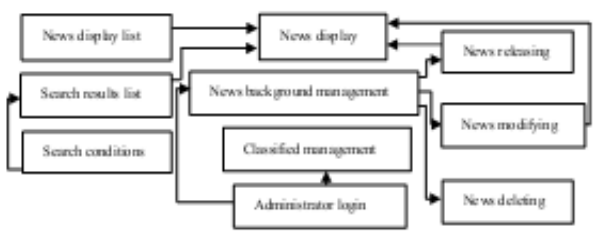

Fig. 2: Structure framework of laboratory news module

\subsection{Experiment teaching}

Experiment teaching module consists of 
teaching idea, laboratory distribution and experiment items. Experiment items introduce the different items separately for the different curriculum. Each item includes experimental principle, experimental equipment, experimental procedure and data processing sub-module. The experiment teaching module structure is shown in Fig. 3.

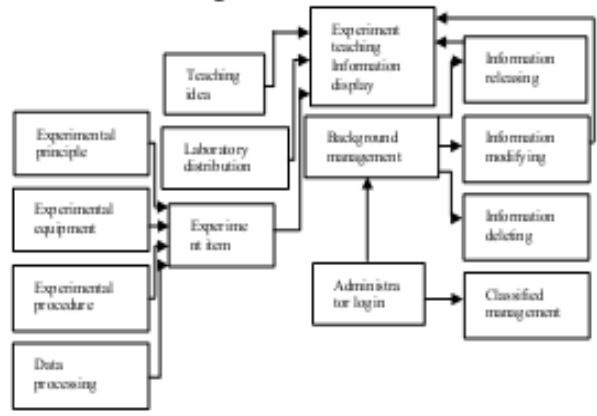

Fig. 3: Structure framework of experiment teaching module

\subsection{Online answering}

Browsers can have a dialogue with the experimental tutor during the learning process through online answering module. By online answering, not only the students and teachers can communicate well to solve problems, but also they can view the other students' questions and answers. Online answering module structure framework is shown in Fig. 4.

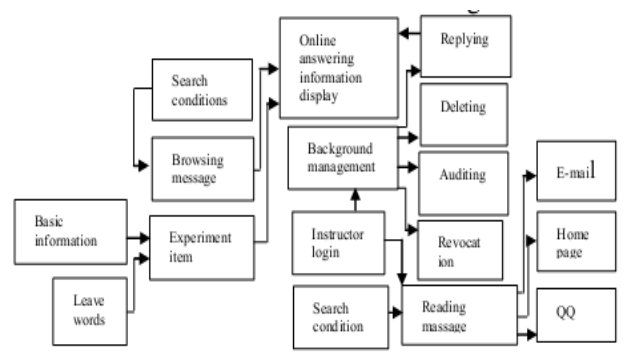

Fig. 4: Structure framework of online answering module

\subsection{Background management}

Background management is the module to maintain the entire website by administrator. The structure framework of the background management module is shown as Fig. 5.

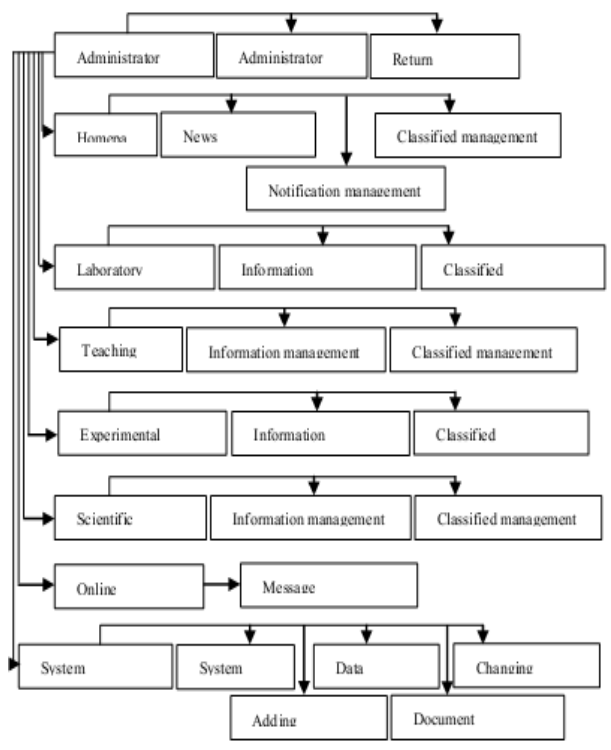

Fig. 5: Structure framework of background management module

\section{Design of the database}

Using the Microsoft Access to create the database, the database name is syjx.mdb. This database is designed four data tables as follows [6].

- Tab. 1 is the teacher information to save the teachers and administrators' information.

- Tab.2 is the experiment information to save the course experiment item information.

- Tab.3 is the massage information to save the student massage.

- Tab.4 is the reply information to save the teachers' reply.

Tab.1: Teacher information

\begin{tabular}{l|l|l}
\hline \multicolumn{1}{c|}{ Head } & \multicolumn{1}{|c|}{ Explanation } & \multicolumn{1}{c}{ Type (length) } \\
\hline ID & Teacher number & $\operatorname{Int}(4)$ \\
UserName & User name & Char(20) \\
PWD & Pass word & Char(20) \\
Purview & purview & Char(10) \\
\hline
\end{tabular}


Tab. 2: Experiment information

\begin{tabular}{l|l|l}
\hline \multicolumn{1}{c|}{ Head } & \multicolumn{1}{|c}{ Explanation } & Type (length) \\
\hline ID & Courses number & $\operatorname{Int}(4)$ \\
Name & Courses name & Char(10) \\
Course Type & Course Type & $\operatorname{int}(6)$ \\
Profession & Profession & $\operatorname{int}(6)$ \\
Ename & Experiment name & Char(20) \\
Eyl & Experimental principle & Char(100) \\
Eyq & Experimental equipment & Char(100) \\
Ebz & Experimental procedure & Char(500) \\
Ecl & Data processing & Char(500) \\
\hline
\end{tabular}

Tab. 3: Massage information

\begin{tabular}{l|l|l}
\hline \multicolumn{1}{c|}{ Head } & \multicolumn{1}{|c|}{ Explanation } & \multicolumn{1}{c}{ Type (length) } \\
\hline ID & Reply number & $\operatorname{Int}(4)$ \\
TopicID & Topic number & $\operatorname{Int}(4)$ \\
Content & Reply content & ntext(16) \\
Reply time & Reply time & smalldatetime(4 \\
Author & Author & ) \\
Face & Face & nchar(50) \\
\hline
\end{tabular}

Tab. 4: Reply information

\begin{tabular}{l|l|l}
\hline \multicolumn{1}{c|}{ Head } & \multicolumn{1}{|c|}{ Explanation } & \multicolumn{1}{c}{ Type (length) } \\
\hline ID & Title number & $\operatorname{Int}(4)$ \\
Title & Title & nchar(225) \\
Content & Content & ntext(500) \\
Create time & Create time & smalldatetime(4) \\
Author & Author & nchar(50) \\
Face & Face & nchar(15) \\
Email & Email & nchar(50) \\
QQ & QQ number & Char(10) \\
Homepage & Homepage & Char(100) \\
Hit & The frequency of hit & Int(4) \\
Reply & The frequency of reply & Int(4) \\
ReplyT & The time of reply & smalldatetime(4) \\
\hline
\end{tabular}

\section{Module realization}

Using JavaScript language edits ASP program, programming method conforms to the principles of JavaScript. For the length of the article is limited, there only lists the programs of the administrator login screen of background management system.

$\langle \%$ abcfifisd $=2 \%\rangle$

$<!--\#$ include file $=$ "../Include/DBClass.inc1.asp"-->

$<!--\#$ include file $="$. ./Include/MD5.asp" -->

$<\%$ Dim Position, aAction, Errmsg, aUserID, aPassWD, aRePassWD, aLevel

Position $=$ GetSafeInt(Trim(Request("Position")),0)

If Position < 1 Then

$$
\begin{aligned}
& \text { Errmsg = "" } \\
& \text { Position }=1
\end{aligned}
$$

Else

Errmsg $="\langle$ font color $=\# F F 0000\rangle\langle b\rangle$ You do not login or stay too long, please re-login $!\langle/ \mathrm{b}\rangle\langle\langle$ font $\rangle "$

End If

aAction $=$ GetSafeStr(Request.Form("action"))
If aAction = "true" Then Dim Server_v1, Server_v2

Server_v1=Cstr(Request.ServerVariables("HTTP_REFERER"))

Server_v2=Cstr(Request.ServerVariables("SERVER_NAME"))

aUserID = Trim(Request("a_userid"))

aPassWD = Trim(Request("a_pwd"))

aRePassWD = Trim(Request("a_rpwd"))

If aUserID $<>" "$ And aPassWD $<>" "$ and aRePassWD $<>" ~ "$

Then

If aRePassWD = CStr(Session("pSN")) Then

sSql $=$ "SELECT $*$ FROM Admin WHERE A_UserID

="'\&aUserID\&"'"

oRs. Open sSql, oConn, 1, 1

If Not oRs.Eof Then

If oRs("A_PWD") = md5(aPassWD) Then' Login Successful!

aLevel = oRs("A_Level") Session("Sys_Admin") = aUserID

Session("Sys_Level") = aLevel

Response.Write If Position $=2$ Then

$"<$ script language ='JavaScript' $>$ top.document.location.reload ()$;</$ script $>"$

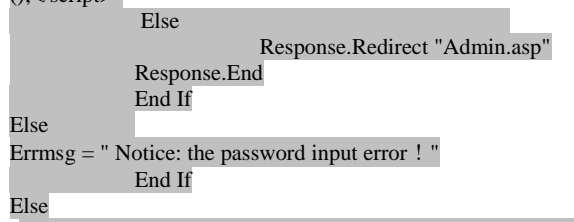

Errmsg = "Notice: The user name input error or does not exist!

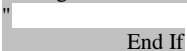

oRs.Clos

Set oRs $=$ Nothing

Else

Errmsg = "Notice: Verify Code input errors ! " End If

Else

Errmsg = "Notice: User name or password or Verify Code can not be empty !"

End If
$\%>$
$\langle$ ETML $>$
$\langle$ HEAD $>$

\section{$\langle\mathrm{HEAD}>$}

$\langle$ TITLE $\rangle\langle \%=$ Homepage Title\% $\%-$-Management Center

Administrator Login $\langle/$ TITLE $>$

$<$ meta http-equiv="Content-Type" content="text/html; charset=gb2312">

$\langle$ script language $=$ "JavaScript" $\rangle$

$<!-$

function window_onload() \{ document.mail.a_userid.focus();

function cancel()

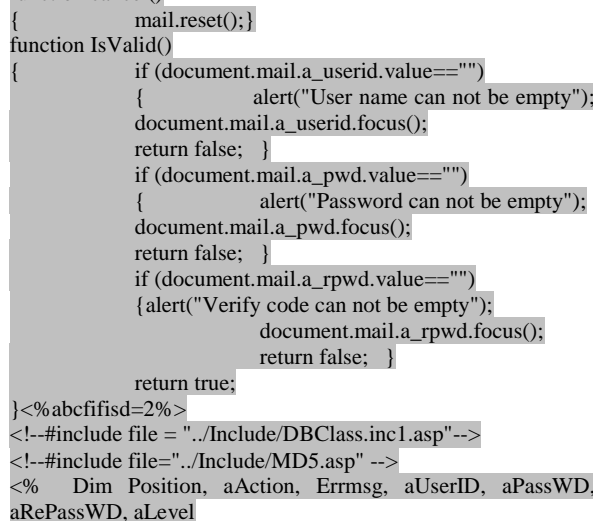
aRePassWD, aLevel 


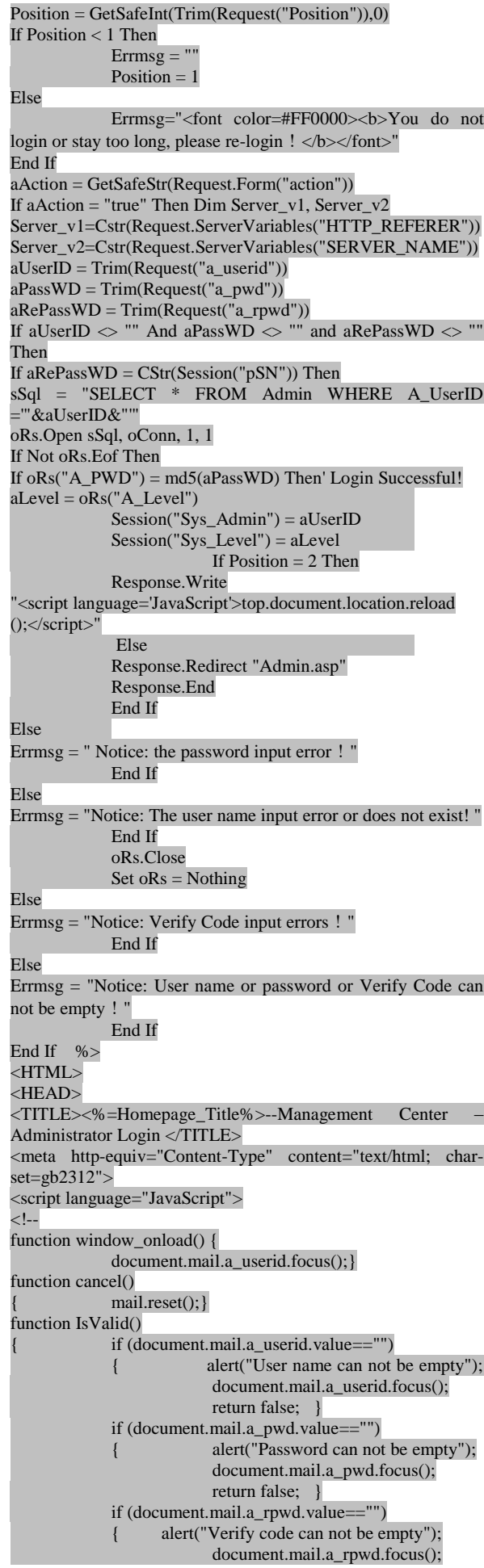

\section{return true;}

\section{Acknowledgment}

This subject supported by Heilongjiang Province higher education reform project and Northeast Forestry University reform project.

\section{Summary}

Experiment teaching method based on WEB makes a breakthrough at the time and space limitation for working, improving learning efficiency, cutting the operation costs. It is an effective means to improve the resources utilization ratio.

\section{References}

[1] Paul Davard. Development of the $\mathrm{Ne}$ twork Technologies. Communieatio-n \& Computer. vol.14, pp.35-36,2010.

[2] http://lwcool.com/lw/newsfile/2008/4 /6/200846_lwcool_14974.html.

[3] Li Chen, Xiao ShengLing, You ShiZhou. Development of the Packaging Engineering Experimental Teaching Platform Based on Interaction Design Concept. Proceedings of 2010 Second International Conference on MultiMedia and Information Technology, pp.162-166, 2010.

[4] Xiao Shengling; Li Chen; Zhang Peijian. Research on Scheme Design and Construction Method of Forest Engineering Network Virtual Laboratory. Forest Engineering.Vol. 25, NO.3, pp.125-128, 2010.

[5] Cao YanLong. ASP/ASP.NET practiceal engineering cases selected of database development. Beijing: Posts \& Telecom Press, pp.236-245, 2011.

[6] Xu Chunyan. Construction and Realization on the Professional Technology Demonstration School Website with the ASP Technology.ChangChun: Jilin University,pp.11-12,2011. 